\title{
Multiscale Characteristics of Human Sleep EEG Time Series
}

\author{
In-Ho Song ${ }^{1,2}$, In-Young Kim² ${ }^{2}$ Doo-Soo Lee ${ }^{1}$, and Sun I. Kim² \\ ${ }^{1}$ Department of Electrical and Computer Engineering, Hanyang University \\ ${ }^{2}$ Department of Biomedical Engineering, College of Medicine, Hanyang University \\ Haengdang-dong, Seongdong-ku, Seoul, 133-791, South Korea \\ sunkim@hanyang.ac.kr
}

\begin{abstract}
We investigated the complexity of fluctuations in human sleep electroencephalograms (EEGs) by multifractals. We used human sleep EEG time series taken from normal, healthy subjects during the four stages of sleep and rapid eye movement (REM) sleep. Our findings showed that the fluctuation dynamics in human sleep EEGs could be adequately described by a set of scales and characterized by multifractals. Multifractal formalism, based on the wavelet transform modulus maxima, appears to be a good method for characterizing EEG dynamics.
\end{abstract}

\section{Introduction}

Since Babloyantz et al. reported that human sleep EEG signals had chaotic attractors during stage 2 and stage 3 sleep, nonlinear dynamic methods based on the concept of chaos have been used to analyze sleep EEG signals [1]. Fell et al. analyzed sleep EEG signals using the largest Lyapunov exponent (LLE) during sleep stages 1-4 and rapid eye movement (REM) sleep, and found significant differences in the values of LLE between the four stages and REM sleep [2] 3]. Kobayashi et al. found significant decrements in the mean correlation dimensions of EEGs from being awake to stage 3 sleep, and an increment during REM sleep 4 . However, these methods require a relatively large number of stationary data points to obtain reliable results [5] 6]. Indeed, it is difficult to meet this requirement in sleep EEGs, as well as in practical physiological systems [5] 6]. Recent research suggests that EEGs can be characterized in some situations by a single dominant timescale, indicating timescale invariance and a $1 / f$ fractal structure [7] 8]. However, many physiological time series fluctuate in a complex manner and are inhomogeneous, suggesting that different parts of the signal have different scaling properties [9] [10]. A new method is needed to analyze sleep EEG signals, and one potential candidate is multifractal analysis based on wavelet transform modulus maxima (WTMM), which is a suitable method for obtaining information about dynamic systems without requiring a relatively large number of stationary data points [10] [1].

The aim of this study was to investigate the possibility that human sleep EEG signals can be characterized by a multifractal spectrum using WTMM. To 
investigate whether physiological brain states according to sleep stages affect the phenomenon of multifractality in human sleep EEG dynamics, we compared the multifractal properties of sleep EEG signals obtained during the four sleep stages and REM sleep. Additionally, we used a surrogate data method to assess whether human sleep EEGs have long-range correlations.

\section{Materials and Methods}

\subsection{Materials}

The sleep EEG data files used in this study were selected from the sleep-EDF database consisting of physiological data obtained from four subjects with horizontal EOG, EEG, submental-EMG, oronasal airflow, etc. Fpz-Cz EEGs were sampled at $100 \mathrm{~Hz}, 12$ bits per sample [12. Sleep stages were scored according to the standard criteria of Rechtschaffen and Kales on $30 \mathrm{~s}$ epochs [12]. The file names used were sc4002e0, sc4012e0, sc4102e0 and sc4112e0. From these, 150 segments (30 awake, 30 stage 1, 30 stage 2, 30 slow wave sleep (SWS), and 30 REM) with a duration of $30 \mathrm{~s}$ (3000 points) were used. Band-pass filter settings were $0.53-40 \mathrm{~Hz}(12 \mathrm{~dB} /$ octave).

\subsection{WTMM-Based Multifractal Formalism}

As stated above, many physiological time series, including human sleep EEGs, have inhomogeneity and fluctuate in an irregular manner. To characterize sleep EEG signals and to extract local Hurst exponents from them, we used a WTMMbased multifractal formalism. The wavelet transform of sleep EEG signal, $f(x)$, is defined as

$$
T_{\psi}[f]\left(x_{0}, a\right)=\frac{1}{a} \int_{-\infty}^{\infty} f(x) \psi\left(\frac{x-x_{0}}{a}\right) d x
$$

where $x_{0}$ is the position parameter, $a$ is the scale parameter, and $\psi(t)$ is the mother wavelet function [11. In this study, the third derivative of the Gaussian function was used as the analyzing wavelet.

A partition function $Z_{q}(a)$ was defined as the sum of the power of order $q$ of the local maxima of $\left|T_{\psi}[f]\left(x_{0}, a\right)\right|$ at scale $a[11]$.

$$
Z_{q}(a)=\sum_{l \in L(a)}\left|T_{\psi}[f]\left(x_{0 l}(a), a\right)\right|^{q}
$$

where $L(a)$ is the set of the maxima lines $l$ existing at the scale $a$, and $x_{0 l}(a)$ is the position, at the scale $a$, of the maximum belonging to the line $l$.

For small scales, it was expressed as

$$
Z_{q}(a) \sim a^{\tau(q)}
$$

where $\tau(q)$ is is the multifractal spectrum. For monofractal signals, $\tau(q)$ is a linear function: $\tau(q)=q h(q)-1$, where $h(q)=d \tau(q) / d q=$ constant, and is 
the global Hurst exponent 9] [10. For multifractal signals, $\tau(q)$ is a nonlinear function and $h(q)=d \tau(q) / d q=$ not constant [9] [10]. For positive values of $q$, $Z_{q}(a)$ characterizes the scaling of the large fluctuations and strong singularities, whereas for negative values of $q, Z_{q}(a)$ characterizes the scaling of the small fluctuations and weak singularities.

Using a Legendre transform, the singularity spectrum $D(h)$ can be expressed as [9] [10] [11.

$$
D(h)=q \frac{d \tau(q)}{d q}-\tau(q)
$$

$D(h)$ can quantify the statistical properties of the different subsets characterized by different exponents, $h$. Nonzero $D(h)$ and $h=0.5$ imply that the fluctuations in signal exhibit uncorrelated behavior. $h>0.5$ corresponds to correlated behavior, while values of $h$ in the range $0<h<0.5$ imply anticorrelated behavior [9] [10.

\subsection{Surrogate Time Series and Statistical Analysis}

To assess the presence of a long-range correlation in the human sleep EEG time series, we generated a surrogate time series by shuffling and integrating the human sleep EEG signal. The surrogate time series that was generated lost the long-range correlation; however, the distribution of the original human sleep EEG signal was preserved. To examine the differences between the mean values of the local Hurst exponents with maximum $D(h)$ for all sleep stages, one-way analysis of variance (ANOVA) and post-hoc analyses were performed. A pairedsample $t$ test was used to compare the mean values of the original data and the surrogate data for each sleep stage. The significance level was 0.05 .

\section{Results}

Multifractal spectra and singularity spectra were computed using the WTMM for the four sleep stages, REM sleep, and surrogate data. Ensemble averaged multifractal spectra, $\tau(q)$, for the four sleep stages, REM sleep and surrogate data were computed, and each singularity spectrum $D(h)$ was computed through a Legendre transform from the ensemble-averaged $\tau(q)$. Figure 1 shows the ensembleaveraged multifractal spectra for the awake stage and REM sleep with surrogate data. The spectra for the awake stage and its surrogate data are shown in the first panel of Fig. 1(a), and the spectra for REM sleep and its surrogate data are shown in the second panel of Fig. 1(b). The two shapes of $\tau(q)$ for the awake stage and its surrogate data, and for REM sleep and its surrogate data were different $(p<0.0001)$ in the range $q>3$.

Likewise, Figure. 2 shows the ensemble-averaged multifractal spectra for the other sleep stages and their surrogate data. The spectra for stage 1 sleep and its surrogate data are shown in the first panel of Fig. 2(a). The second panel 
of Fig. 2(b) shows the spectra for stage 2 sleep and its surrogate data, and the third panel of Fig. 2(c) shows the spectra for SWS and its surrogate data. The two shapes of $\tau(q)$ between stage 1 sleep and its surrogate data, between stage 2 sleep and its surrogate data, and between SWS and its surrogate data, were all different $(p<0.0001)$ for $q(-10<q<+10)$.
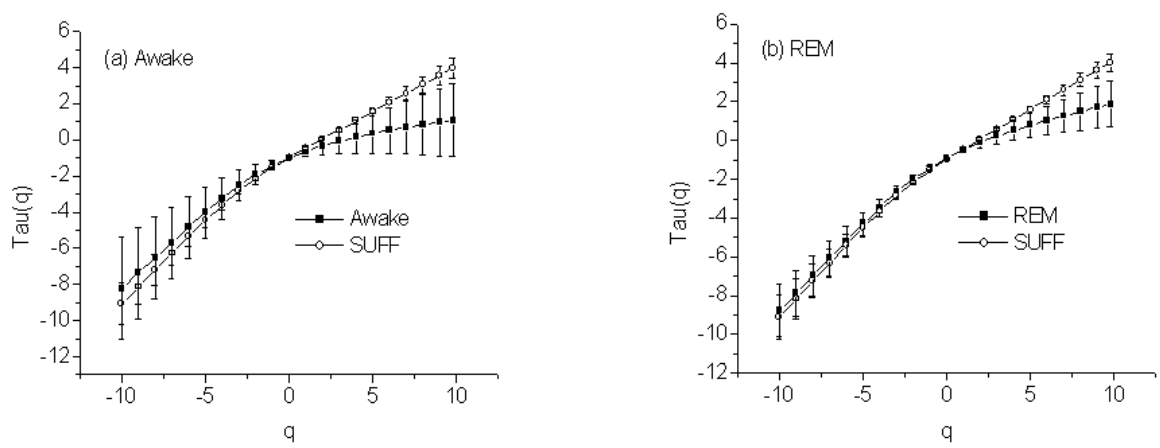

Fig. 1. Ensemble-averaged multifractal spectra for the awake stage and REM sleep with surrogate data. (a) $\tau(q)$ for the awake stage and its surrogate data. (b) $\tau(q)$ for REM sleep and its surrogate data.
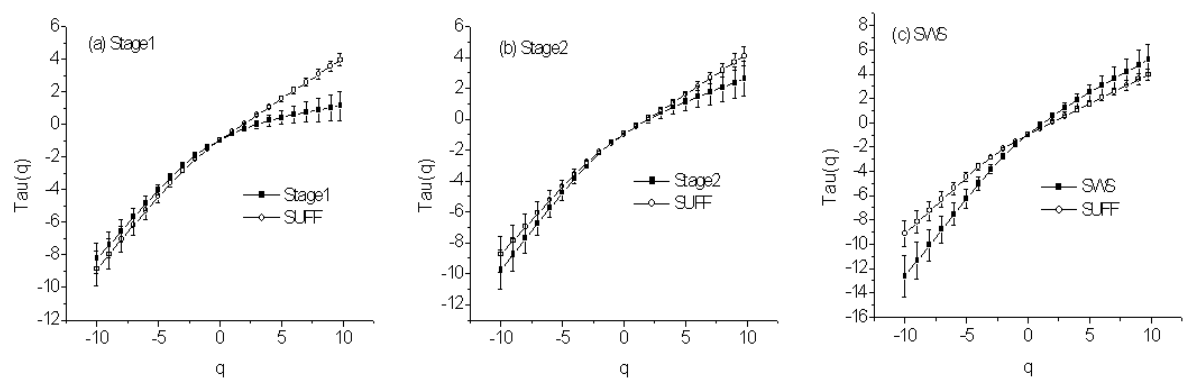

Fig. 2. Ensemble-averaged multifractal spectra for the other sleep stages and their surrogate data. (a) $\tau(q)$ for stage 1 sleep and its surrogate data. (b) $\tau(q)$ for stage 2 sleep and its surrogate data. (c) $\tau(q)$ for SWS and its surrogate data.

The singularity spectra for the four sleep stages, REM sleep and their surrogate data are shown in Fig. 3 and 4 . The two shapes of $D(h)$ between each sleep stage and its surrogate data, and between REM sleep and its surrogate data, were significantly different. The range of local Hurst exponents with $D(h)$ greater than 0.75 was $0.24<h<1$ for each sleep stage and for REM sleep. However, the local Hurst exponents in the range $0.48<h<0.7$ corresponded to a $D(h)$ greater than 0.75 for the surrogate data.

Singularity spectra for the awake stage and its surrogate data are shown in the first panel of Fig. 3(a), and singularity spectra for REM sleep and its surrogate 

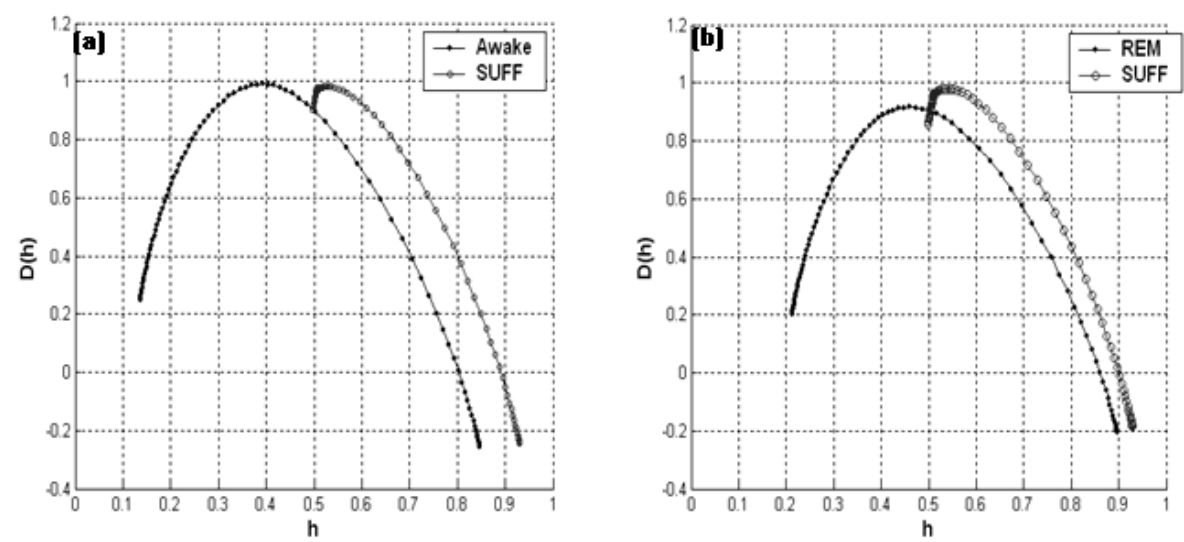

Fig. 3. (a) Sigularity spectra, $D(h)$, for the awake stage and its surrogate data. (b) Singularity spectra for REM sleep and its surrogate data. The different shape of $D(h)$ for the surrogate data reflects different fluatuations associated with long-range correlation.
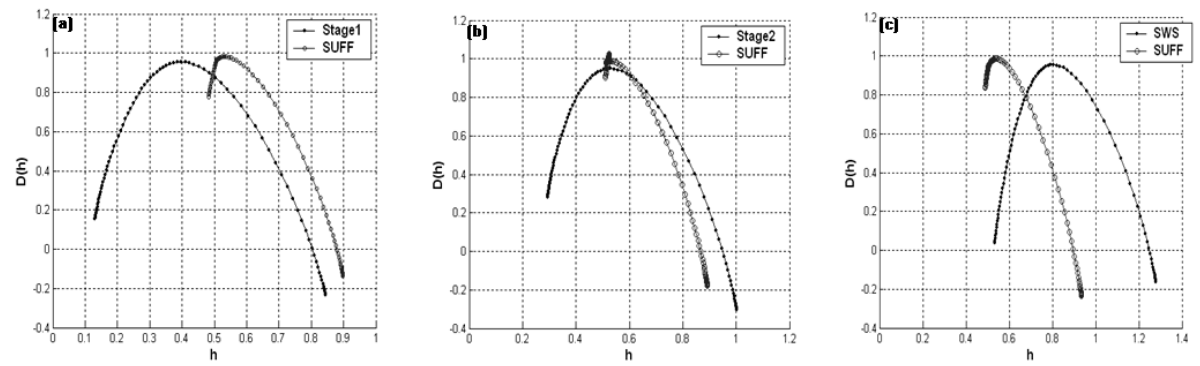

Fig. 4. (a) Sigularity spectra, $D(h)$, for stage 1 sleep and its surrogate data. (b) Singularity spectra for stage 2 sleep and its surrogate data. (c) Singularity spectra for SWS and its surrogate data. The different shape of $D(h)$ for the surrogate data reflects different fluatuations associated with long-range correlation.

data are shown in the second panel of Fig. 3(b). The mean values of local Hurst exponents with maximum $D(h)$ for the awake stage and for REM sleep were 0.39 and 0.46 , respectively. The singularity spectra for stage 1 sleep and its surrogate data are shown in the first panel of Fig. 4(a). The second panel of Fig. 廿(b) shows the singularity spectra for stage 2 sleep and its surrogate data, and the third panel of Fig. 4(c) shows the singularity spectra for SWS and its surrogate data. The mean values of local Hurst exponents with maximum $D(h)$ for stage 1 , stage 2 , and SWS were $0.39,0.52$ and 0.80 , respectively. The results of the ANOVA are shown in Table 1 . 


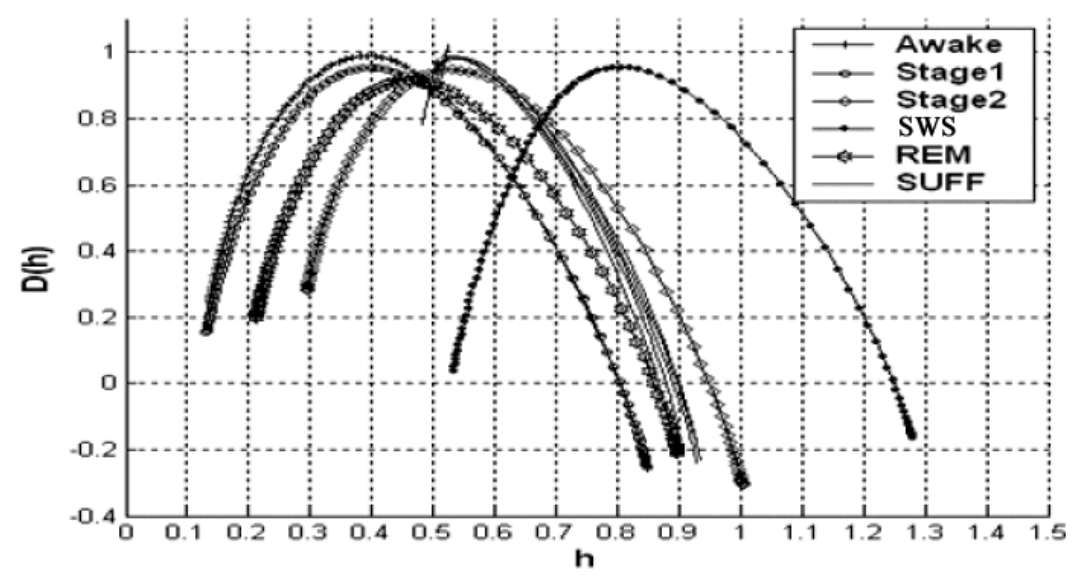

Fig. 5. Comparison of singularity spectra for each sleep stage, REM sleep and the surrogate data

Table 1. Comparison of local Hurst exponents with maximum $D(h)$. The results are presented as mean values \pm SD. ANOVA was performed, followed by Sidak post hoc analysis.

\begin{tabular}{ccc}
\hline Sleep stages and REM sleep & Mean \pm Std & Sidak post hoc analysis \\
\hline a) Awake & $0.39 \pm 0.29$ & $\mathrm{a}<\mathrm{c}(p<0.01) ; \mathrm{a}<\mathrm{d}(p<0.001)$ \\
b) Stage 1 & $0.39 \pm 0.05$ & $\mathrm{~b}<\mathrm{c}(p<0.01) ; \mathrm{b}<\mathrm{d}(p<0.0001)$ \\
c) Stage 2 & $0.52 \pm 0.08$ & $\mathrm{c}>\mathrm{a}, \mathrm{b}(p<0.01) ; \mathrm{c}<\mathrm{d}(p<0.001)$ \\
d) SWS & $0.80 \pm 0.11$ & $\mathrm{~d}>\mathrm{a}, \mathrm{b}, \mathrm{c}, \mathrm{e}(p<0.0001)$ \\
e) REM & $0.46 \pm 0.12$ & $\mathrm{e}<\mathrm{d}(p<0.0001)$ \\
\hline
\end{tabular}

\section{Discussion and Conclusion}

Figure 1 and 2 show the shapes of $\tau(q)$ for the four sleep stages and REM sleep were nonlinear, and Fig. 5 shows the shapes of $D(h)$ for the four sleep stages and REM sleep were broad, indicating that human sleep EEGs could not be characterized as monofractal and are therefore multifractal.

The subsets characterized by local Hurst exponents in the range $0.24<h<0.63$ were statistically dominant for the awake stage, stage 1 sleep, and REM sleep. We found that the dynamics of human sleep EEGs taken during the awake stage, stage 1 sleep, and REM sleep exhibited anticorrelated behaviors, indicating that large values are more likely to be followed by small values and vice versa. Statistically dominant subsets were characterized by local Hurst exponents of $0.38<h<0.71$ for stage 2 sleep. We found that the dynamics of human sleep EEGs during stage 2 sleep exhibited anticorrelated, correlated, and uncorrelated behaviors. For SWS, the subsets characterized by local Hurst exponents in the range $0.66<h<1$ were statistically dominant. We found that 
the dynamics of human sleep EEGs during SWS exhibited correlated behaviors. Therefore, we suggest that physiological brain states according to sleep stages affect the phenomenon of multifractality in human sleep EEG dynamics.

ANOVA showed a significant difference in the mean value of the local Hurst exponents with maximum $D(h)(F(4,145)=35.17, p<0.0001)$. Post-hoc analysis showed that the mean value of the local Hurst exponents with maximum $D(h)$ for SWS differed significantly $(p<0.0001)$ from that of all other sleep stages, including REM sleep. However, there was no significant difference in the mean values of the local Hurst exponents with maximum $D(h)$ for the awake stage, stage 1 sleep, and REM sleep. The mean value of the local Hurst exponents with maximum $D(h)$ for stage 2 sleep did not differ from that for REM sleep; however, it differed significantly $(p<0.01)$ from that for all other sleep stages. These findings indicate that as the sleep stage changes from stage 1 to SWS, the dynamics of human sleep EEGs change from anticorrelated dynamics to correlated dynamics. These findings are consistent with previous studies that show the activity of the human brain may become low as SWS sleep occurs.

The results observed after the surrogate test showed significant changes in the shape of $D(h)$ and in the values of the local Hurst exponents with maximum $D(h)$ for the four sleep stages and the REM sleep. The paired-sample $t$ test showed significant differences between the awake stage and the surrogate data, and between the REM sleep and the surrogate data, for the range $q>3$. These findings indicate that the scaling of the small fluctuations and weak singularities in human sleep EEGs during the awake stage and during REM sleep is similar to that in a simple random walk. However, the scaling of the large fluctuations and strong singularities in human sleep EEGs during the awake stage and REM sleep differ significantly from that in a simple random walk. The results of the paired-sample $t$ test showed significant differences between stage 1 sleep and the surrogate data, between stage 2 sleep and the surrogate data, and between SWS and the surrogate data, for all $q(-10<q<+10)$. These findings indicate that there are different fluctuations and different characteristics for the singularities between stage 1 sleep and the surrogate data, between stage 2 sleep and the surrogate data, and between SWS and the surrogate data. Therefore, we suggest that human sleep EEGs taken during the four sleep stages and REM sleep have long-range correlations because the surrogate data preserve the distributions of the original time series and remove long-range correlations.

In summary, we investigated the possibility that human sleep EEGs exhibit higher complexity than $1 / f$ scaling. Our findings show the existence of inhomogeneities and multifractalities in human sleep EEGs during the four sleep stages and REM sleep. We found that human sleep EEGs taken during the awake stage and during REM sleep exhibited anticorrelated behaviors and had longrange correlations, whereas SWS EEGs exhibited correlated behaviors and had long-range correlations. We suggest that the complexity of human sleep EEGs can better be described by a set of scales rather than a single dominant scale.

Acknowlegements. This study was supported by a Nano-bio technology development project, Ministry of Science \& Technology, Republic of Korea (2005-01249). 


\section{References}

1. Acharya U, R., Faust, O., Kannathal, N., Chua, T., Laxminaryan, S.: Non-linear analysis of EEG signals at various sleep stages. Comput. Meth. Prog. Bio. 80 (2005) 37-45

2. Fell, J., Röschke, J., Beckmann, P.: Deterministic chaos and the first positive Lyapunov expoent: a nonlinear analysis of the human electroencephalogram during sleep. Boil. Cybernet. 69 (1993) 139-146

3. Fell, J., Röschke, J., Mann, K., Schäffner, C.: Discrimination of sleep stages: a comparison between spectral and nonlinear EEG measures. Electroencephalogr. Clin. Neurophysio. 98 (1996) 401-410

4. Kobayashi, T., Misaki,K., Nakagawa, H., Madokoro, S., Ihara, H., Tsuda, K., Umezawa, Y., Murayama, j., Isaki, K.: Non-linear analysis of the sleep EEG. Psychiatry Clin. Neurosci. 53 (1999) 159-161

5. Thomasson, N., Hoeppner,T.J., Webber Jr, C.L., Zbilut, J.P.: Recurrence quantification in epileptic EEGs. Phys. Lett. A. 279. (2001) 94-101

6. Song, I.H., Lee, D.S., Kim, Sun.I.: Recurrence quantification analysis of sleep electroencephalogram in sleep apnea syndrome in humans. Neurosci. Lett. 366 (2004) $148-153$

7. Watters, P.A.: Time-invariant long-range correlations in electroencephalogram dynamics. Int. J. Sys. Sci 31 (2000) 819-825

8. Linkenkaer-Hansen, K., Nikulin, V.V., Palva, S., Ilmoniemi, R.J., Palva, J.M.: Long-range temporal correlations and scaling behavior in human brain oscillations. J. Neurosci. 21 (2001) 1370-1377

9. Ivanov, P.Ch., Amaral, Luís A.N., Goldberger, A.L., Havlin, S., Rosenblum, M.G., Struzik, Z.R., Stanley, H.E.: Multifractality in human heartbeat dynamics. Nature. 399 (1999) 461-465

10. Ivanov, P.Ch., Amaral, Luís A.N., Goldberger, A.L., Havlin, S., Rosenblum, M.G., Stanley, H.E., Struzik, Z.R.: From $1 / f$ noise to multifractal cascades in heartbeat dynamics. Chaos, 11 (2001) 641-652

11. Muzy, J.F., Bacry, E., Arneodo, A.: The multifractal formalism revisited with wavelet. Int. J. Bifurc. chaos. 4 (1994) 245-302

12. Mourtazaev, M.S., Kemp, B., Zwinderman, A.H., Kamphuisen, A.C.H.: Age and gender affect different characteristics of slow waves in the sleep EEG. Sleep $\mathbf{1 8}$ (1995) 557-564 